\title{
SCHUR CONVEXITY OF DUAL FORM OF THE COMPLETE SYMMETRIC FUNCTION
}

\author{
KONGSHENG ZHANG AND HUANNAN SHI
}

Abstract. In this paper, the Schur-convexity, the Schur-geometric-convexity and the Schur-harmonicconvexity of dual form of the complete symmetric function are investigated. As consequences, some new inequalities are established via majorilization theory.

Mathematics subject classification (2010): 26B25, 05E05.

Keywords and phrases: complete symmetric function, dual form, Schur-convexity, Schur-geometricconvexity, Schur-harmonic-convexity.

\section{REFERENCES}

[1] Y.-M. ChU And Y.-P. Lv, The Schur harmonic convexity of the Hamy symmetric function and its applications, J. Inequal. Appl., 2009, Art. ID. 838529, 10 pages.

[2] Y.-M. CHU AND T.-C. SUN, The Schur harmonic convexity for a class of sym- metric functions, Acta Math. Sci. Ser. B Engl. Ed., 30 (2010), 1501-1506.

[3] Y.-M. CHU, G.-D. WANG AND X.-H. ZHANG, The Schur multiplicative and harmonic convexities of the complete symmetric function, Math. Nachr., 284 (2011), 653-663.

[4] Y.-M. CHU AND W.-F. XIA, Necessary and sufficient conditions for the Schur harmonic convexity of the generalized Muirhead mean, Proc. A. Razmadze Math. Inst., 152 (2010), 19-27.

[5] Y.-M. CHU, W.-F. XIA AND X.-H. ZHANG, The Schur concavity, Schur multiplicative and harmonic convexities of the second dual form of the Hamy symmetric function with applications, J. Multivariate Anal., 105 (2012), 412-421.

[6] Y.-M. ChU, W.-F. XIA AND T.-H. ZHAO, Schur convexity for a class of symmetric functions, Sci. China Math., 53 2010, 465-474.

[7] Y.-M. CHU AND X.-M. ZHANG, Necessary and sufficient conditions such that extended mean values are Schur-convex or Schur-concave, J. Math. Kyoto Univ., 48 (2008), 229-238.

[8] Y.-M. Chu, X.-M. Zhang AND G.-D. WANG, The Schur geometrical convexity of the extended mean values, J. Conv. Anal., 15 (2008), 707-718.

[9] K.-Z. GuAn, Schur-convexity of the complete symmetric function, Math. Inequal. Appl., 9 (2006), $567-576$.

[10] K.-Z. Guan, Some properties of a class of symmetric functions, J. Math. Anal. Appl., 33 (2007), $670-680$.

[11] K.-Z. GuAn, The Hamy symmetric function and its generalization, Math. Inequal. Appl., 9 (2006), 797-805.

[12] K.-Z. GUAN AND J.-H. SHEN, Schur-convexity for a class of symmetric function and its applications, Math. Inequal. Appl., 9 (2006), 199-210.

[13] W.-D. JIANG, Some properties of dual form of the Hamy's symmetric function, J. Math. Inequal., 1 (2007), 117-125.

[14] B.-Y. LONG AND Y.-M. CHU, The Schur convexity and inequalities for a class of symmetric functions, Acta Math. Sci. Ser. A Chin. Ed., 32 (2012), 80-89.

[15] A. W. Marshall And I. OlKin, Inequalities: Theory of Majorization and Its Applications, Academic Press, New York, 1979.

[16] J.-X. Meng, Y.-M. Chu And X.-M. TAng, The Schur-harmonic-convexity of dual form of the Hamy symmetric function, Matematicki Vesnik, 62 (2010), 37-46. 
[17] I. SCHUR, Über eine Klasse von Mittelbildungen mit Anwendungen auf die Determinantentheorie, Sitzunsber Berlin Math Ges, 22 (1923), 9-20.

[18] J. N. Whiteley, Some inequalities concerning symmetric forms, Mathematika, 5 (1958), 47-49.

[19] W.-F. Xia And Y.-M. CHU, On Schur-convexity of some symmetric functions, J. Inequal. Appl., 2010, Art. ID. 543250, 12 pages.

[20] W.-F. XIA AND Y.-M. CHU, Schur convexity and Schur multiplicative convexity for a class of symmetric functions with applications, Ukrainian Math., 61 (2009), 1541-1555.

[21] W.-F. XIA AND Y.-M. CHU, Schur-convexity for a class of symmetric functions and its applications, J. Inequal. Appl., 2009, Art. ID. 493759, 15 pages.

[22] W.-F. XIA AND Y.-M. CHU, Schur convexity with respect to a class of symmetric functions and their applications, Bull. Math. Anal. Appl., 3 (2011), 84-96.

[23] W.-F. XIA AND Y.-M. ChU, The Schur convexity of Gini mean values in the sense of harmonic mean, Acta Math. Sci. Ser. B, Engl. Ed., 31 (2011), 1103-1112.

[24] W.-F. XIA, Y.-M. CHU AND G.-D. WANG, Necessary and sufficient conditions for the Schur harmonic convexity or concavity of the extended mean values, Rev. Un. Mat. Argentina, 52 (2011), 121132.

[25] W.-F. XIA, G.-D. WANG AND Y.-M. CHU, Schur convexity and inequalities for a class of symmetric functions, Int. J. Pure Appl. Math., 58 (2010), 435-452.

[26] X.-M. Zhang, Geometrically Convex Functions, An'hui University Press, Hefei, 2004 (in Chinese).

[27] X.-M. Zhang, S-Geometric convexity of a function involving Maclaurin's elementary symmetric mean, J. Inequal. Pure. Appl. Math., Vol. 8, 2007, Article 51, 6 pages. 